Research Articles: Behavioral/Cognitive

\title{
Sensory-Tactile Functional Mapping and Use- Associated Structural Variation of the Human Female Genital Representation Field
}

https://doi.org/10.1523/JNEUROSCI.1081-21.2021

Cite as: J. Neurosci 2021; 10.1523/JNEUROSCI.1081-21.2021

Received: 10 June 2021

Revised: 26 October 2021

Accepted: 29 November 2021

This Early Release article has been peer-reviewed and accepted, but has not been through the composition and copyediting processes. The final version may differ slightly in style or formatting and will contain links to any extended data.

Alerts: Sign up at www.jneurosci.org/alerts to receive customized email alerts when the fully formatted version of this article is published.

Copyright ( 2021 Knop et al.

This is an open-access article distributed under the terms of the Creative Commons Attribution 4.0 International license, which permits unrestricted use, distribution and reproduction in any medium provided that the original work is properly attributed. 
Pages: 29; Tables: 2; Figures: 5

Word Counts: Abstract: 150; Introduction: 648; Discussion: 1503

\section{Sensory-Tactile Functional Mapping and Use-Associated Structural Variation of the Human Female}

\section{Genital Representation Field}

Andrea J.J. Knop, M.S. ${ }^{1 a}$, Stephanie Spengler, Ph.D. ${ }^{1 a}$, Carsten Bogler, Ph.D. ${ }^{1 b, 2}$, Carina Forster, M.S. ${ }^{3}$, Michael Brecht, Ph.D. ${ }^{2,4 a}$, John-Dylan Haynes, Ph.D. ${ }^{1 \mathrm{~b}, 2,4 b^{*}}, \&$ Christine Heim, Ph.D. ${ }^{1 \mathrm{a}, 5^{*}}$

${ }^{1}$ Charité - Universitätsmedizin Berlin, corporate member of Freie Universität Berlin and Humboldt-Universität zu Berlin, ${ }^{\mathrm{a}}$ Institute of Medical Psychology, ${ }^{\mathrm{b}}$ Berlin Center for Advanced Neuroimaging, Berlin, Germany;

${ }^{2}$ Bernstein Center for Computational Neuroscience, Berlin, Germany; ${ }^{3}$ Max Planck Institute for Human Cognitive and Brain Sciences, Leipzig, Germany; ${ }^{4}$ Humboldt-Universität zu Berlin, ${ }^{\mathrm{a}}$ Department of Biology, ${ }^{b}$ Department of Psychology, Berlin, Germany; ${ }^{5}$ Department of Biobehavioral Health, The Pennsylvania State University, University Park, PA, USA.

Running Title:

\section{LOCALIZATION AND VARIATION OF FEMALE GENITAL FIELD}

*Shared last authors.

Corresponding Author: Christine Heim, Ph.D., Professor and Chair of the Institute of Medical Psychology, Charité - Universitätsmedizin Berlin, Luisenstr. 57, 10117 Berlin, Germany. Tel: +49 (0) 30450529 222; Fax: +49(0) 30450529 990; Email: christine.heim@ charite.de

Acknowledgement: Funded by a NeuroCure Cluster of Excellence (Deutsche Forschungsgemeinschaft EXC 2049) intramural innovation grant to $\mathrm{CH}$ and MB, the Max Planck School of Cognition to $\mathrm{CH}$ and JDH, and a scholarship from the Einstein Center for Neuroscience Berlin to AJJK. We thank Kristina Sandt and Frieda Born for assistance.

Conflict of Interest Statement: The authors declare no competing financial interests. 


\section{ABSTRACT (150)}

The precise location of the human female genital representation field in the primary somatosensory cortex (S1) is controversial and its capacity for use-associated structural variation as a function of sexual behavior remains unknown. We used an fMRI-compatible sensory-tactile stimulation paradigm to functionally map the location of the female genital representation field in 20 adult women. Neural response to tactile stimulation of the clitoral region (versus right hand) identified individually-diverse focal bilateral activations in dorsolateral areas of S1 (BA1-BA3) in alignment with anatomical location. We next used cortical surface analyses to assess structural thickness across the 10 individually most activated vertices per hemisphere for each woman. We show that frequency of sexual intercourse within 12 months is correlated with structural thickness of the individually-mapped left genital field. Our results provide a precise functional localization of the female genital field and provide support for use-associated structural variation of the human genital cortex.

\section{SIGNIFCANCE (120)}

We provide a precise location of the human female genital field in the somatosensory cortex and, for the first time, provide evidence in support of structural variation of the human genital field in association with frequency of genital contact. Our study represents a significant methodological advance by individually mapping genital fields for structural analyses. On a secondary level, our results suggest that any study investigating changes in the human genital field must map the field individually to achieve sufficient precision. Our results pave the way for future research into the plasticity of the human genital cortex as a function of normal or adverse experience as well as changes in pathological conditions, i.e. sexual dysfunction, sexual deviation or sexual risktaking behavior. 


\section{INTRODUCTION (648)}

The precise location of the female genital representation field in the primary somatosensory cortex (S1) is still a matter of contention (Di Noto et al., 2013; Cazala et al., 2015). Furthermore, the capacity of the human genital representation field for use-associated structural plasticity has never been studied.

In their first presentation of the somatosensory homunculus, Penfield and Rasmussen (1950) placed the male genital field below the foot in the mesial part of S1. This non-somatotopic location of the genital field was supported by results demonstrating functional activations in the mesial wall of the paracentral lobe in response to electrical stimulation of the dorsal penile nerve in males (Allison et al., 1996; Nakagawa et al., 1998; Mäkelä et al., 2003) and manual-tactile clitoral, vaginal, and cervical self-stimulation in females (Komisaruk et al., 2011). Other studies provided evidence for a somatotopically-ordered representation of the genital field adjacent to the hip and knee areas by demonstrating activations in dorsolateral regions of the postcentral gyrus in response to electrical stimulation of the dorsal clitoral nerve (Michels et al., 2010) or partner-delivered manual stimulation of the clitoris in females (Georgiadis et al., 2006, 2009), as well as sensory-tactile brushing of the penile shaft in males (Kell et al., 2005). These latter results are in line with evidence from rodent studies that localize the rat genital cortex in somatotopic order and bilateral symmetry (Lenschow et al., 2016; Lenschow and Brecht, 2018).

The mode of stimulation used in functional mapping studies may contribute to heterogeneous results concerning the location of the genital field in humans. Specifically, electrical stimulation is not equivalent to sensory touch and elicits less focal responses (Pratt et al., 1980; Forss et al., 1994). Self- or partner-delivered manual stimulation includes touching of areas adjacent to the genitals and elicits sexual arousal that may confound neural response (Georgiadis et al., 2006, 2009, 2010; Komisaruk et al., 2011). The only study using a focal sensory-tactile non-arousing stimulation paradigm in the form of soft brushing of the penile shaft was limited to men and does not inform about female genital field location (Kell et al., 2005). Indeed, no study to date has functionally mapped the female genital field in humans using a magnetic resonance imaging (MRI)compatible focal sensory-tactile non-arousing stimulation paradigm, contrasting neural response to sensory stimulation of the clitoris against sensory stimulation of a control region. 
Commensurate with the fact that the precise location of the genital field remains controversial, there is no evidence regarding its capacity for structural change in association with use in humans. It is well-established that the human brain has substantial capacity for plasticity as a function of experience (e.g., Draganski and May, 2008). Use-dependent structural reorganization of human S1 has been observed after deprivation of afferent input due to limb amputation (Elbert et al., 1994; Flor et al., 1995; Knecht, 1998) or peripheral nerve lesion (Henderson et al., 2011). Whether or not the human genital field is capable to structurally adapt to its normal use is entirely unknown. Recent evidence suggests that the developing rat genital cortex expands with genital stimulation, facilitating puberty (Lenschow et al., 2017; Sigl-Glöckner et al., 2019).

We here combine the investigation of the location of the female genital field with the question of structural variation of this field as a function of sexual behavior, considering the important issue of individual variability: 1) We provide a precise localization the human female genital representation field by using a focal sensory-tactile non-arousing stimulation paradigm during fMRI to contrast neural response of stimulation of the clitoral region versus the right hand. 2) We use individually-mapped genital fields based on the 10 most activated vertices per hemisphere for each woman and assess structural thickness in the individually-mapped field using cortical surface analysis. 3) We show that thickness of the individually-mapped genital field varies with the frequency of sexual intercourse in the past 12 months, compatible with use-associated plasticity.

\section{MATERIALS AND METHODS}

\section{Sample}

We recruited 25 adult healthy women aged 18 to 45 years. General exclusion criteria applied to select women were lifetime or current psychiatric disorders, exposure to childhood abuse or neglect (including sexual abuse), neurological disorders, physical disease, central nervous system or urogenital surgery, psychotropic medication within six months, sexually transmitted disease, sexual disorders (including sexual anxiety, discontent or dysfunction or dissociation during sexual activity), past or current pregnancy, and current menstruation. Exclusionary conditions were assessed using clinician-administered interviews and standard questionnaires (Oldfield, 1971; Hahlweg, 1996, Anon, 2000; Berner et al., 2004; Kühner et al., 2007; BrenkFranz and Strauß, 2011; Hansen et al., 2012; Klinitzke et al., 2012; Hoyer et al., 2015; Müller, 2016). Women were screened for contraindications of MRI scanning. Of the 25 women recruited into the study, 20 women were included in the analyses. Five women were excluded because the experimental procedure (i.e., genital stimulation paradigm) was not successful. 


\section{Procedure}

Women underwent a standardized study visit at the Institute of Medical Psychology and the Berlin Center for Advanced Neuroimaging, both at Charité - Universitätsmedizin Berlin. During the visit, women underwent all study procedures, including interviews and questionnaires for demographics and exclusionary conditions. To localize the genital representation field in S1, women underwent 1) fMRI scanning during sensory-tactile stimulation of the clitoris versus dorsum of the right hand, 2) structural MRI to assess thickness of the individually mapped genital field, and 3) a detailed sexual history to assess frequency of sexual intercourse, i.e. genital sensory touch, in the past year and lifetime for the assessment of use-dependent plasticity of the individually mapped genital field. The study was approved by the institutional ethics committee and was conducted in accordance with the Declaration of Helsinki. Written informed consent of the participants was obtained.

\section{MRI Acquisition}

Structural MRI was performed using a 3.0 T Siemens Tim Trio MRI scanner (Siemens Medical System, Erlangen, Germany) with a standard 12-channel head coil. Two 1-mm (Sanchez Panchuelo et al., 2018) isotropic $\mathrm{T} 1$ anatomical scans were acquired in the sagittal plane using the magnetization-prepared rapid gradient echo sequence $($ MPRAGE; TR/TE $=2530 / 4.94 \mathrm{~ms}$, slice number $=176)$. Structural MRI acquisition took 2 x 6:03 minutes. Functional MRI scans were obtained using a T2*-weighted echoplanar image pulse sequence (EPI; $\mathrm{TR} / \mathrm{TE}=2000 / 30 \mathrm{~ms}$, slice number $=32$, voxel size $=3 \times 3 \times 3 \mathrm{~mm}$, slice gap $=0.75 \mathrm{~mm})$. The functional imaging paradigm comprised 4 scanning blocks with a duration of 5:36 minutes, respectively.

\section{Experimental Design and Statistical Analysis}

\section{Sensory-Tactile Stimulation Paradigm}

We developed an MRI-compatible sensory-tactile stimulation paradigm that allows for administering a defined focal sensory stimulus to the clitoral region (see Figure 1). The stimulation was administered using a non-invasive air-controlled oscillating membrane with a compression of approximately 0.1 bar. Women were asked to place the membrane below the mons pubis on the clitoral area above standardized disposable underwear. The sensory-tactile device was fixed with elastic tape and a flexible Velcro belt. Sensory-tactile stimulation of the dorsum of the right hand was used as a control condition, given that the S1 representations of the dermatomes of the genital region and the hand are well distinguishable (Roux et al., 2018). 
The paradigm was performed in an ABBA versus $\mathrm{BAAB}$ block design with stimulation of either the clitoral region (A) or the dorsum of the right hand (B) interspersed with 10-second periods of no stimulation. Each of the four runs started with a period of no stimulation and included a total of eight clitoral and eight dorsum manus stimulation phases. The order of these phases was fixed and counterbalanced between women. Synchronization of the trigger pulses from the MRI scanner and the timing of the stimulation was controlled using Presentation (Neurobehavioral Systems Inc., Albany, CA, USA). During the sensory-tactile stimulation, subjects were asked to fixate a cross on a screen. One woman completed only 3 runs.

Pleasantness and sexual arousal during clitoral stimulation were assessed after each run using a 7-point visual analogue scale. Subjects were instructed to use a fiberoptic response box, indicating changes in pleasantness and sexual arousal. We then computed combined ratings on overall pleasantness and sexual arousal after the scan. We further inquired on the subjective appropriateness of the location of the clitoral membrane during the experiment as well as on sensations in other body parts during clitoral stimulation. There was no evidence for dislocation of the stimulation membrane in the sample.

\section{Localization of Genital Field}

Statistical parametric mapping 12 (SPM12; Wellcome Trust Centre for Neuroimaging, University College London, London, UK) was used to perform functional image analysis in order to localize the genital field in S1. Standard spatial preprocessing of functional images, including realignment and co-registration to T1 image, was separately performed for each of the four scanning blocks. Data were high-pass filtered with a default cut-off period of 128 seconds to correct for slow drift artifacts. There was no head motion above $3.0 \mathrm{~mm}$ and 3.0 deg of maximal translation and rotation in any direction throughout a scanning block.

After standard spatial preprocessing, functional MRI data were analyzed using a general linear model (GLM). The two within-subject conditions of interest (10 seconds of either clitoral or hand stimulation alternating with 10 seconds of rest) were modelled using a boxcar function convolved with a canonical hemodynamic response function (HRF). Activation maps were calculated with t-tests for contrasts between the two regressors of the design matrix, resulting in individual patterns of neural activation in response to clitoral versus hand stimulation. We identified an activated region in SI for each participant at $\mathrm{p}<0.001$ without correction or $\mathrm{p}<0.05$ with family-wise error (FWE) correction for multiple comparisons. Individual neural activations were overlaid onto co-registered anatomical scans and saved as individual regions of interest (ROI) 
for the left and right hemisphere, respectively. Individual ROI was multiplied with the t-score map corresponding to the individual contrast image to delineate the most activated vertices within the individually defined ROI. We purposely did not perform spatial normalization to a standard stereotaxic space (Montreal Neurological Institute EPI template; MNI) or smoothing of the images to allow for subsequent cortical thickness analyses within the individually mapped ROI in native space of the anatomical images, as needed for the usedependent plasticity analyses (see below). To determine variability of the location of the genital field and hand representation in S1 between women, coordinates of peak neural activation were transformed in MNI space. Barycentre and dispersion across individually mapped fields were computed by averaging individual coordinates in MNI space.

To additionally localize the genital representation field in $\mathrm{S} 1$ on the group level, a random effects general linear model was estimated across subjects. For this, individual contrast maps were spatially normalized to a standard MNI template and resampled to an isotropic spatial resolution of $3 \times 3 \times 3 \mathrm{~mm}$. Furthermore, data were spatially smoothed with a $6 \mathrm{~mm}$ full-width at half-maximum isotropic Gaussian kernel. Whole brain grouplevel analysis with t-tests contrasting neural response to the two with-subject factors genital stimulation versus stimulation of the dorsum of the right hand was thresholded at $\mathrm{p}<0.05$ with FWE-correction for multiple comparisons. Coordinates of the group-based neural activation reflecting the genital field are given in standard MNI space.

These statistical analyses and figures were computed using Matlab (Mathworks, Version 9.6.)

\section{Anatomical Image Segmentation and Surface-Based Morphometry (CAT12)}

Automated image segmentation included (1) spatial registration (affine registration to tissue probability map); (2) initial SPM Unified Segmentation and skull stripping; (3) local intensity transformation to reduce tissue inhomogeneities (Local Adaptive Segmentation (Dahnke et al., 2012)); (4) volumetric segmentation of grey matter (GM), white matter (WM), and cerebrospinal fluid (CSF), as well as GM-WM and GM-CSF, providing a more accurate segmentation (Tohka et al., 2004); (5) Spatial normalization / Dartel registration (Ashburner, 2007), (6) central surface estimation (Projection-Based Thickness Method; Dahnke et al., 2013), (7) topology correction (Yotter et al., 2011a); (8) surface inflation (spherical mapping; Yotter et al., 2011b) and spherical atlas registration (resampling; Yotter et al., 2011c), and default merging of hemispheres. 


\section{Thickness of Individually Mapped Genital Field}

The Computational Anatomy Toolbox 12 (CAT12; Christian Gaser, Structural Brain Mapping Group, Jena University Hospital, Jena, Germany) for SPM12 was used to perform cortical surface-based morphometry (SBM) of the anatomical scans. Image segmentation was conducted using an automated standard procedure (see Supplement). The individually defined ROIs for the clitoris and the dorsum of the right hand were separately mapped onto individual native space cortical surfaces of the left and right hemisphere. After cortical surface registration, mean thickness of the 10 functionally most active vertices within the individually mapped ROIs (as described above) was separately calculated for each hemisphere in each woman. Cortical thickness at each vertex was calculated as part of central surface estimation (Dahnke et al., 2013), describing the closest distance between the inner surface (white matter/grey matter boundary) and the outer surface (grey matter/pial boundary) at each vertex of the tessellated brain surface (Fischl and Dale, 2000; Dahnke et al., 2013).

\section{Use-Associated Structural Variation of the Genital Field}

We assessed mean frequency of sexual intercourse per week using a standardized biographic questionnaire to quantify sexual intercourse within the past 12 months and in 5 year ranges since the onset of the first sexual genital contact. As noted above, we excluded sexual anxiety, discontent or dysfunction as well as dissociation during intercourse using established questionnaires (Anon, 2000; Berner et al., 2004; Brenk-Franz and Strauß, 2011; Hansen et al., 2012; Hoyer et al., 2015; Müller, 2016). To associate cortical thickness measures of the individually mapped genital field with data on sexual behavior, we correlated individual cortical thickness with the mean frequency of sexual intercourse per week within the past 12 months. We further correlated cortical thickness of the individually mapped genital field with the frequency of sexual intercourse estimated across a longer time period since the first onset of sexual contact. As we calculated one correlation per hemisphere, we did apply a Bonferroni-correction for multiple comparisons to the results $\left(\alpha_{\text {corr }}=.025\right)$. Using partial correlation analyses, we used age, years since onset of sexual contact, and whole brain cortical thickness as covariates to control for effects of these variables on genital field cortical thickness. Furthermore, correlations and partial correlations between left-hemispheric cortical thickness of the representation field of the right hand and frequency of sexual intercourse for either time window were calculated to confirm for region-specificity of use-associated variation. These statistical analyses and figures were computed using R Project for Statistical Computing (R Core Team, Version 4.0.2) and IBM SPSS Statistics (IBM, Version 27). 


\section{RESULTS}

\section{Demographic and Behavioral Data}

Demographic and behavioral data are presented in Table 1. Mean age of the sample was 23.10 years $(S D=4.35)$. The majority of women was of European descent, had a higher education, were heterosexual, lived in a monogamous partnership, and were right-handed. Seven women were on oral contraceptives. MR scans were distributed across menstrual cycle phase. Mean frequency of sexual intercourse in the past 12 months was reported to have been 1.91 times per week $(S D=1.30)$. Mean frequency of sexual intercourse since the onset of sexual contact was reported to have been 1.46 times per week $(S D=0.93)$. Importantly, behavioral data obtained during the sensory-tactile stimulation paradigm confirmed that the stimulation was not unpleasant and neither overly pleasant nor overly sexually arousing.

\section{Functional Mapping of the Female Genital Field: Neural Response to Sensory-Tactile Stimulation} Sensory-tactile stimulation of the clitoral region (relative to right hand) induced significant focal neural activations in S1. Sixteen women exhibited bilateral neural activations in S1. For four women, a significant activation was found in either the right or the left hemisphere only. Table 2 delineates individual MNI coordinates with the respective $\mathrm{p}$ value thresholds and $\mathrm{T}$-scores of the sensory foci for clitoral stimulation. Individual focal neural activations occurred in Brodmann Areas 1, 2, and 3a / 3b (BA1-3) of the postcentral gyrus for all women. Within BA1-3, there was distinctive individual variability of the precise location of the neural activation in response to stimulation of the clitoral region. Figure 2 shows the individual localization of the clitoral somatosensory representation in normalized stereotaxic coordinates (MNI space).

We next mapped the individual representation of the right hand for use in subsequent cortical thickness analyses. Sensory-tactile stimulation of the dorsum of the right hand (relative to clitoral region) induced significant contralateral focal neural activations in S1. Table 2 delineates individual MNI coordinates with the respective $\mathrm{p}$ value thresholds and $\mathrm{T}$-scores of the sensory foci for the stimulation of the right hand. Individual focal neural activations occurred in BA1-3 of the postcentral gyrus, with individual variability of the precise location of the neural activation. Figure 3 shows the individual localization of the somatosensory representation of the hand in normalized stereotaxic coordinates (MNI space) for the left hemisphere. There was no significant effect of handedness on functional activation of the hand representation. Of note, the location of the representation field of the clitoris and the representation field of the hand was somatotopically-ordered for each woman and commensurate with anatomical location. 
When analyzed at the group level across all women, general linear models revealed significant symmetric dorsolateral neural activations in S1 in response to stimulation of the clitoris (relative to hand) in both hemispheres (left hemisphere: $\mathrm{x}=-18, \mathrm{y}=-34, \mathrm{z}=74 ; T=7.72, p_{\text {FWE-corr }}=0.024$; right hemisphere: $\mathrm{x}=18, \mathrm{y}=-$ $\left.40, \mathrm{z}=68 ; T=10.26, p_{\text {FWE-corr }}<0.0001\right)$. Of note, no other significant neural activations were observed at the group level in response to the stimulation of the clitoral region, suggesting that the stimulation paradigm specifically targeted the genital field and was not overly arousing. Figure 4 shows normalized stereotaxic coordinates (MNI space) for the group location mapped onto the cortical surface.

\section{Use-Associated Structural Variation of the Female Genital Field: Surface-Based Morphometry}

We mapped individual ROIs for the genital field (representing the 10 most activated vertices per hemisphere during clitoral stimulation) onto native cortical surfaces for each subject and estimated cortical thickness of the individual genital representation field (for individual data, see Table 2). Partial correlation analysis controlling for age, years since onset of sexual contract, and whole brain cortical thickness revealed a significant positive correlation between cortical thickness of the individually-mapped left-hemispheric genital field and the frequency of sexual intercourse within the past 12 months $(r=.701, p=.004$; corrected $p<.05)$. Similarly, longer-term frequency of sexual intercourse estimated since the onset of sexual contact was significantly correlated with thickness of the individually-mapped left-hemispheric genital field in a partial correlation analysis $(r=.538, p=.039)$. Partial correlation analyses between cortical thickness of the righthemispheric genital field and frequency of sexual intercourse did not reveal any significant effects, suggesting lateralized use-associated structural variation. Figure 5 shows scatterplots of left genital field thickness against frequency of sexual intercourse for the past 12 months and frequency of sexual intercourse since the onset of sexual contact, plotted as residuals corrected for covariates. Of note, menstrual cycle phase was not significantly associated with thickness of the genital field.

To confirm the specificity of this effect, we mapped individual ROIs for the representation of the hand (representing the 10 most activated vertices in the left hemisphere in response to stimulation of the right hand) onto native cortical surfaces for each woman and estimated cortical thickness of the individual representation field of the hand (for individual data, see Table 2). Importantly, cortical thickness of the hand representation was not significantly associated with frequency of sexual intercourse at either time window, with or without correction for the effects of covariates, reflecting a highly specific use-dependent effect for the sensory field involved in the specific behavior. 


\section{DISCUSSION}

We present novel evidence on the precise location of the female genital representation field and its capacity for use-associated structural variation. Using functional mapping during sensory-tactile stimulation of the clitoral region, we show focal bilateral neural activations within the dorsolateral postcentral gyrus in S1. We show that the individual location of peak neural activations in response to clitoral stimulation varies considerably between women. We applied cortical surface analysis to the individually-mapped ROI to compute structural thickness of the genital field. Correlating the individually-mapped morphological data with behavioral data on sexual contact, we provide first evidence that thickness of the genital field varies as a function of frequency of genital intercourse in the past 12 months and lifetime, in line with use-associated plasticity.

Our results are noteworthy in several ways. To localize the female genital field, we measured neural response in a tactile-sensory stimulation paradigm that delivers a physiologically valid stimulus as opposed to a previous study using electrical stimulation of the clitoris (Michels et al., 2010). Furthermore, our tactile-sensory stimulation paradigm did not involve touching of body parts adjacent to the clitoris nor did it induce marked sexual arousal as opposed to previous studies using self- or partner-delivered stimulation (Georgiadis et al., 2006, 2009, 2010; Komisaruk et al., 2011). The sole other study that used a sensory-tactile non-arousing stimulation paradigm to localize the genital field was limited to males (Kell et al., 2005). Our stimulation paradigm induced focal targeted neural activations, without inducing neural activation in other brain regions, at comparatively (Kell et al., 2005; Michels et al., 2010) high levels of statistical significance without using somatosensory template masks. Therefore, our data provide unequivocal information about the location of the female genital field and represent a significant methodological advance compared to previous studies that yielded conflicting results (Georgiadis et al., 2006, 2009; Michels et al., 2010; Komisaruk et al., 2011), likely due to confounding factors inherent to stimulation paradigms used in these studies (Pratt et al., 1980; Forss et al., 1994). On a group level, the mean location of the female genital field in the dorsolateral postcentral gyrus, identified in our study, corresponds with the location reported in two of the previous studies in females using electrical (Michels et al., 2010) or partner-delivered manual stimulation (Georgiadis et al., 2006) as well as with the location reported for males in the above-referenced study using sensory-tactile stimulation in males (Kell et al., 2005). Our results confirm a somatotopically-ordered representation of the female clitoris, adjacent to the representation of the hips and upper legs and commensurate with anatomical location, and disprove displaced location in the mesial wall of the precentral lobe. Our results provide independent confirmation for the revision (Kell et al., 2005) of the original homunculus (Penfield and Rasmussen, 1950) and extend the validity of the 
revised homunculus to women. Our results confirm a bilateral somatosensory representation of the anatomically centered clitoris, in line with histological mapping data on the localization and bilateral representation of the rat genital cortex (Lenschow et al., 2016; Lauer et al., 2017; Lenschow and Brecht, 2018).

Our results suggest profound variability of the individual location of the genital field within the dorsolateral part of S1 with individual peak activations clearly deviating from the group mean. This means that any study looking at structural variation of the genital field as a function of certain conditions, such as sexual behavior, sexual abuse or sexual dysfunction, must necessarily implement individual mapping of the genital field and compute data, i.e. cortical thickness, on an individual level. Clearly, only by using individually-mapped ROIs, such studies yield precise reliable surface-based parameters for association with specific conditions.

We computed data on structural thickness of the genital field in individually-mapped ROIs, based on the 10 most activated vertices per hemisphere for each woman. We show that individual thickness of the left genital field associates with frequency of sexual intercourse. The association was stronger for genital intercourse within the past 12 months. While less pronounced, the association was significant for lifetime genital contact. Frequency of genital intercourse was not associated with thickness of the representation field of the right hand nor with thickness of the entire cortical mantle, confirming a specific association between genital touch and genital field thickness. This is compatible with the idea that the female genital field has capacity for structural plasticity depending on its use, commensurate with the general "use-it-or-lose-it" principle of experiencedependent plasticity (eg., Hebb, 1947; Elbert and Rockstroh, 2004; Draganski and May, 2008). While injury- or use-dependent plasticity in the human somatosensory cortex has been reported (Elbert et al., 1994, 1995; Flor et al., 1995; Foell et al., 2014), our results are the first to document structural variation of genital field thickness associated with more or less frequent normative use. Our results are in line with findings from animal studies showing that genital brushing during puberty resulted in lateral expansion of the rat and mouse genital cortex (Lenschow et al., 2017; Sigl-Glöckner et al., 2019). Cortical plasticity serves to enhance the efficiency of processing of behaviorally-relevant inputs and represents an adaptive response (Trachtenberg et al., 2002; Markham and Greenough, 2004; Feldman and Brecht, 2005; May, 2011). In an earlier study, we observed decreased thickness of the genital cortex after exposure to childhood sexual abuse, suggesting that highly aversive and developmentally inappropriate sexual stimulation may limit somatosensory representation to decrease processing of detrimental input (Heim et al., 2013). 
Several mechanisms might contribute to dynamic use-associated structural plasticity of the genital field. Structural thickening of the mature cortex as a function of use most likely reflects formation of new synapses by axonal sprouting, dendritic arborization, and dendritic spine growth rather than induction of new neurons through neurogenesis (Markham and Greenough, 2004; Feldman and Brecht, 2005; Feldman, 2009; May, 2011). There is substantial evidence on the central role of glutamatergic synapses in mediating plasticity, reflecting rapid components of N-methyl-D-aspartate (NMDA) receptor-dependent long-term potentiation (LTP) and depression (LTD) (Buonomano and Merzenich, 1998; Feldman, 2009). Another mechanism contributing to useassociated structural plasticity may involve alterations in glial-cell mediated myelination (Timmler and Simons, 2019). While oligodendrogenesis is rare (Yeung et al., 2019), the presence of large numbers of pre-myelinating oligodendrocytes in the human cortex may enable adaptive myelination to adapt conduction velocity to functional demand (Gibson et al., 2014). Future studies in humans should use novel imaging tools that allow for assessing cortical myelin density (Amunts and Zilles, 2015) to study genital field plasticity. Further, neural activation in response to somatosensory stimulation depends on axonal input from the thalamus (Feldman, 2009). When removing afferent somatosensory input from the thalamus, dendritic spine numbers of somatosensory cortical neurons attenuate (Lendvai et al., 2000). When exposing rats to genital touch or sexual contact during puberty, invading thalamo-cortical afferents promote the expansion of the female genital cortex (Lenschow et al., 2016). Future studies on genital field plasticity should therefore include assessments of thalamo-cortical connectivity and myelination.

It must be noted that use-associated variation of structural thickness of the female genital field in our study was limited to the left hemisphere. This lateralized effect is puzzling given that the neural representation of the clitoris is bilateral. Left-hemispheric dominance of neural plasticity has been reported for learning-dependent structural change after coordination and motor skill training (Draganski et al., 2004; Taubert et al., 2010; Rogge et al., 2018). Such lateralized plasticity may reflect hemispheric specialization (Serrien et al., 2006). In the above referenced study (Heim et al., 2013), thinning of the genital field after sexual abuse was limited to the left hemisphere. While we cannot comprehensively explain these findings, one plausible mechanism may involve lateralized limbic-cortical modulation of sensory afferent inputs into the genital field, leading to unilateral associations of sexual behavior with genital field morphology.

While our localization of the female genital field was experimental in nature, our investigation of the capacity of the genital field for structural variation as a function of genital contact was cross-sectional and relied 
on retrospective self-report of genital intercourse. Our results align with the general principle of an association between frequency of genital intercourse and structural variation, albeit the direction of effect is a matter of discussion. It is conceivable that thickness of the genital field may drive frequency of sexual intercourse. Results from animal models provide causal that clitoral stimulation drives genital field thickness (Lenschow et al., 2016; Lenschow and Brecht, 2018). Future prospective studies or studies exploiting quasi-experimental conditions, such as induction of behavior change during sexual therapy, are needed to establish causality.

In conclusion, we provide an unequivocal localization of the female genital field in S1 and support for use-associated plasticity of the human genital field. On a secondary level, our findings support the notion that studies investigating change of the human genital field must map the field individually. Our results pave the way for future research into the plasticity of the human genital field as a function of normal or adverse experience as well as genital field structure, function and plasticity in pathological conditions, such sexual dysfunction, sexual deviation or sexual risk-taking behavior. 
402

403

404

405

406

407

408

409

410

411

412

413

414

415

416

417

418

419

420

\section{AUTHOR CONTRIBUTIONS}

AJJK and $\mathrm{CH}$ wrote the manuscript. JDH, MB, and SS edited the manuscript. $\mathrm{CH}$, JDH, and MB conceived the study and obtained funding. AJJK and SS implemented and conducted the study. CF assisted in data collection. AJJK, CB, and JDH ran the data analyses.

\section{OTHER AFFILIATIONS}

$\mathrm{CH}, \mathrm{JDH}$, and MB are members of the Berlin School of Mind \& Brain, the Einstein Center for Neuroscience Berlin, and the Max Planck School of Cognition. CH and MB are members of the NeuroCure Cluster of Excellence Berlin. CH is member of the Penn State Child Maltreatment Solutions Network. AJJK is affiliated with the Berlin School of Mind and Brain, the Einstein Center for Neuroscience Berlin, and the Max Planck School of Cognition.

\section{DATA AVAILABILITY STATEMENT}

The datasets generated during and/or analyzed during the current study are available from the corresponding author on reasonable request.

\section{CODE ACCESSIBILITY STATEMENT}

Custom MATLAB Code (Version R2018b, MathWorks Inc.) for SPM12 and CAT 12 will be provided upon request. 


\section{REFERENCES}

Allison T, McCarthy G, Luby M, Puce A, Spencer DD (1996) Localization of functional regions of human mesial cortex by somatosensory evoked potential recording and by cortical stimulation. Electroencephalogr Clin Neurophysiol Potentials Sect 100:126-140.

Amunts K, Zilles K (2015) Architectonic mapping of the human brain beyond brodmann. Neuron 88:1086-1107. Anon (2000) The arizona sexual experience scale (asex): reliability and validity. J Sex Marital Ther 26:25-40. Ashburner J (2007) A fast diffeomorphic image registration algorithm. Neuroimage 38:95-113.

Berner M, Kriston L, Zahradnik H-P, Härter M, Rohde A (2004) Überprüfung der gültigkeit und zuverlässigkeit des deutschen female sexual function index (fsfi-d). Geburtshilfe Frauenheilkd 64:293-303.

Brenk-Franz K, Strauß B (2011) Der multidimensionale fragebogen zur sexualität (mfs). Z Für Sex 24:256271.

Buonomano DV, Merzenich MM (1998) Cortical plasticity: from synapses to maps. Annu Rev Neurosci 21:149186.

Cazala F, Vienney N, Stoléru S (2015) The cortical sensory representation of genitalia in women and men: a systematic review. Socioaffective Neurosci Psychol 5:26428.

Dahnke R, Yotter RA, Gaser C (2013) Cortical thickness and central surface estimation. Neuroimage 65:336348.

Dahnke R, Ziegler G, Gaser C (2012) Local adaptive segmentation. http://www.neuro.unijena.de/hbm2012/HBM2012-Dahnke02.pdf

Di Noto PM, Newman L, Wall S, Einstein G (2013) The hermunculus: what is known about the representation of the female body in the brain? Cereb Cortex 23:1005-1013.

Draganski B, Gaser C, Busch V, Schuierer G, Bogdahn U, May A (2004) Changes in grey matter induced by training. Nature 427:311-312.

Draganski B, May A (2008) Training-induced structural changes in the adult human brain. Behav Brain Res 192:137-142.

Elbert T, Flor H, Birbaumer N, Knecht S, Hampson S, Larbig W, Taub E (1994) Extensive reorganization of the somatosensory cortex in adult humans after nervous system injury. NeuroReport 5:2593-2597.

Elbert T, C Pantev, C Wienbruch, B Rockstroh, E Taub (1995) Increased cortical representation of the fingers of the left hand in string players. Science 270(5234):305-7.

Elbert T, Rockstroh B (2004) Reorganization of human cerebral cortex: the range of changes following use and injury. The Neuroscientist 10:129-141. 
Feldman DE (2009) Synaptic mechanisms for plasticity in neocortex. Annu Rev Neurosci 32:33-55.

Feldman DE, Brecht M (2005) Map plasticity in somatosensory cortex. Science 310:810-815.

Fischl B, Dale AM (2000) Measuring the thickness of the human cerebral cortex from magnetic resonance images. Proc Natl Acad Sci 97:11050-11055.

Flor H, Elbert T, Knecht S, Wienbruch C, Pantev C, Birbaumer N, Larbig W, Taub E (1995) Phantom-limb pain as a perceptual correlate of cortical reorganization following arm amputation. Nature 375:482-484.

Foell J, Bekrater-Bodmann R, Diers M, Flor H. Mirror therapy for phantom limb pain: brain changes and the role of body representation. Eur J Pain 2014;18:729-39.

Forss N, Salmelin R, Hari R (1994) Comparison of somatosensory evoked fields to airpuff and electric stimuli. Electroencephalogr Clin Neurophysiol Potentials Sect 92:510-517.

Georgiadis JR, Farrell MJ, Boessen R, Denton DA, Gavrilescu M, Kortekaas R, Renken RJ, Hoogduin JM, Egan GF (2010) Dynamic subcortical blood flow during male sexual activity with ecological validity: a perfusion fmri study. NeuroImage 50:208-216.

Georgiadis JR, Kortekaas R, Kuipers R, Nieuwenburg A, Pruim J, Reinders AATS, Holstege G (2006) Regional cerebral blood flow changes associated with clitorally induced orgasm in healthy women. Eur J Neurosci $24: 3305-3316$

Georgiadis JR, Reinders AATS, Paans AMJ, Renken R, Kortekaas R (2009) Men versus women on sexual brain function: prominent differences during tactile genital stimulation, but not during orgasm. Hum Brain Mapp 30:3089-3101.

Gibson EM, Purger D, Mount CW, Goldstein AK, Lin GL, Wood LS, Inema I, Miller SE, Bieri G, Zuchero JB (2014) Neuronal activity promotes oligodendrogenesis and adaptive myelination in the mammalian brain. Science 344(6183):1252304.

Hahlweg K (1996) Fragebogen zur Partnerschaftsdiagnostik (fdp). Hogrefe Verlag für Psychologie.

Hansen NB, Brown LJ, Tsatkin E, Zelgowski B, Nightingale V (2012) Dissociative experiences during sexual behavior among a sample of adults living with HIV infection and a history of childhood sexual abuse. J Trauma Dissociation 13:345-360.

Hebb DO (1947) The effects of early experience on problem-solving at maturity. Am Psychol 2:306-307.

Heim CM, Mayberg HS, Mletzko T, Nemeroff CB, Pruessner JC (2013) Decreased cortical representation of genital somatosensory field after childhood sexual abuse. Am J Psychiatry 170:616-623. 
Henderson LA, Gustin SM, Macey PM, Wrigley PJ, Siddall PJ (2011) Functional reorganization of the brain in humans following spinal cord injury: evidence for underlying changes in cortical anatomy. J Neurosci 31:2630-2637.

Hoyer J, Klein V, Schierz K, Briken P (2015) Screening für sexuelle funktionsstörungen nach dsm-5. Z Für Sex $28: 36-42$.

Kell CA, von Kriegstein K, Rösler A, Kleinschmidt A, Laufs H (2005) The sensory cortical representation of the human penis: revisiting somatotopy in the male homunculus. J Neurosci 25:5984-5987.

Klinitzke G, Romppel M, Häuser W, Brähler E, Glaesmer H (2012) Die deutsche Version des Childhood Trauma Questionnaire: Psychometrische Eigenschaften in einer bevölkerungsrepräsentativen Stichprobe. PPmP Psychother Psychosom Med Psychol 62:47-51.

Knecht S (1998) Plasticity of plasticity? Changes in the pattern of perceptual correlates of reorganization after amputation. Brain 121:717-724.

Komisaruk BR, Wise N, Frangos E, Liu W, Allen K, Brody S (2011) Women's clitoris, vagina, and cervix mapped on the sensory cortex: fmri evidence. J Sex Med 8:2822-2830.

Kühner C, Bürger C, Keller F, Hautzinger M (2007) Reliabilität und Validität des revidierten BeckDepressionsinventars (BDI-II): Befunde aus deutschsprachigen Stichproben. Nervenarzt 78:651-656.

Lauer SM, Lenschow C, Brecht M (2017) Sexually selected size differences and conserved sexual monomorphism of genital cortex. J Comp Neurol 525:2706-2718.

Lendvai B, Stern EA, Chen B, Svoboda K (2000) Experience-dependent plasticity of dendritic spines in the developing rat barrel cortex in vivo. Nature 404:876-881.

Lenschow C, Brecht M (2018) Physiological and anatomical outputs of rat genital cortex. Cereb Cortex 28:1472-1486.

Lenschow C, Copley S, Gardiner JM, Talbot ZN, Vitenzon A, Brecht M (2016) Sexually monomorphic maps and dimorphic responses in rat genital cortex. Curr Biol 26:106-113.

Lenschow C, Sigl-Glöckner J, Brecht M (2017) Development of rat female genital cortex and control of female puberty by sexual touch kleinfeld d, ed. PLOS Biol 15:e2001283.

Mäkelä JP, Illman M, Jousmäki V, Numminen J, Lehecka M, Salenius S, Forss N, Hari R (2003) Dorsal penile nerve stimulation elicits left-hemisphere dominant activation in the second somatosensory cortex: sII activation by dpn. Hum Brain Mapp 18:90-99.

Markham JA, Greenough WT (2004) Experience-driven brain plasticity: beyond the synapse. Neuron Glia Biol $1: 351$. 
May A (2011) Experience-dependent structural plasticity in the adult human brain. Trends Cogn Sci 15:475482.

Michels L, Mehnert U, Boy S, Schurch B, Kollias S (2010) The somatosensory representation of the human clitoris: an fmri study. NeuroImage 49:177-184.

Müller MJ (2016) Sexual Behaviour Questionnaire - Deutsche Version [Fragebogen]. In Leibniz-Zentrum für Psychologische Information und Dokumentation (ZPID) (Hrsg.)

Nakagawa H, Namima T, Aizawa M, Uchi K, Kaiho Y, Yoshikawa K, Orikasa S, Nakasato N (1998) Somatosensory evoked magnetic fields elicited by dorsal penile, posterior tibial and median nerve stimulation. Electroencephalogr Clin Neurophysiol Potentials Sect 108:57-61.

Oldfield RC (1971) The assessment and analysis of handedness: the edinburgh inventory. Neuropsychologia 9:97-113.

Penfield W, Rasmussen T (1950) The cerebral cortex of man; a clinical study of localization of function.

Pratt H, Politoske D, Starr A (1980) Mechanically and electrically evoked somatosensory potentials in humans: effects of stimulus presentation rate. Electroencephalogr Clin Neurophysiol 49:240-249.

Rogge A-K, Röder B, Zech A, Hötting K (2018) Exercise-induced neuroplasticity: balance training increases cortical thickness in visual and vestibular cortical regions. NeuroImage 179:471-479.

Roux F, Djidjeli I, Durand J (2018) Functional architecture of the somatosensory homunculus detected by electrostimulation. J Physiol 596:941-956.

Sanchez Panchuelo RM, Besle J, Schluppeck D, Humberstone M, Francis S (2018) Somatotopy in the human somatosensory system. Front Hum Neurosci 12:235.

Serrien DJ, Ivry RB, Swinnen SP (2006) Dynamics of hemispheric specialization and integration in the context of motor control. Nat Rev Neurosci 7:160-166.

Sigl-Glöckner J, Maier E, Takahashi N, Sachdev R, Larkum M, Brecht M (2019) Effects of sexual experience and puberty on mouse genital cortex revealed by chronic imaging. Curr Biol 29:3588-3599.e4.

Taubert M, Draganski B, Anwander A, Muller K, Horstmann A, Villringer A, Ragert P (2010) Dynamic properties of human brain structure: learning-related changes in cortical areas and associated fiber connections. J Neurosci 30:11670-11677.

Timmler S, Simons M (2019) Grey matter myelination. Glia 67:2063-2070.

Tohka J, Zijdenbos A, Evans A (2004) Fast and robust parameter estimation for statistical partial volume models in brain MRI. Neuroimage 23:84-97. 
Trachtenberg JT, Chen BE, Knott GW, Feng G, Sanes JR, Welker E, Svoboda K (2002) Long-term in vivo imaging of experience-dependent synaptic plasticity in adult cortex. Nature 420:788-794.

Yeung MS, Djelloul M, Steiner E, Bernard S, Salehpour M, Possnert G, Brundin L, Frisén J (2019) Dynamics of oligodendrocyte generation in multiple sclerosis. Nature 566:538-542.

Yotter RA, Dahnke R, Thompson PM, Gaser C (2011a) Topological correction of brain surface meshes using spherical harmonics. Hum Brain Mapp 32:1109-1124.

Yotter RA, Thompson PM, Gaser C (2011b) Algorithms to improve the reparameterization of spherical mappings of brain surface meshes. J Neuroimaging 21:e134-e147.

Yotter RA, Ziegler G, Thompson PM, Gaser C (2011c) Diffeometric anatomical registration on the surface. HBM 2011 Poster \#628, 2011d. 


\section{FIGURE LEGENDS}

Figure 1. Device for sensory-tactile stimulation of the clitoral region and dorsum of the right hand. The stimulus is delivered via a non-invasive air-controlled oscillating membrane with a compression of approximately 0.1 bar.

Figure 2. Interindividual variability of the genital somatosensory cortex in the MNI space. (A) Bilateral distribution of single subjects' representation of the clitoris in S1. Brodmann classification was based on probabilistic cytoarchitectonic maps (JuBrain Anatomy Toolbox v3.0; Simon Eickhoff, Institut für Neurowissenschaften und Medizin, Forschungszentrum Jülich, Jülich, Germany). Bicolored data points indicate overlapping Brodmann Areas, depending on the z-coordinate in the transverse plane (see part (D)). (B) Detailed distribution over the two hemispheres, respectively. (C) Barycentres of the genital representations (shown in dots) on the left and right hemisphere with amplitude bars representing the dispersion (shown in lines). MNI barycentres of the genital representation on the left hemisphere ( $x=-19.5$ (SE: \pm 2.8 , Range: $-27--15), y=-38$ (SE: \pm 3.6 , Range: $-46--31), z=72$ (SE: \pm 4.3 , Range: $62-80)$ ) and right hemisphere $(x=18.5$ (SE: \pm 4.3 , Range: $-9-27, y=-38$ (SE: \pm 2.8 , Range: $-43--31$ ), $\mathrm{z}=71.5$ (SE: \pm 4.3 , Range: $62-80)$ ). (D) Schematic representation of the anterior parietal areas $\mathrm{BA} 3 \mathrm{a}, \mathrm{BA} 3 \mathrm{~b}, \mathrm{BA} 1$, and $\mathrm{BA} 2$, indicating that all data points lay within the postcentral gyrus based on a probabilistic atlas of human cortical brain areas (Harvard-Oxford macroanatomical atlas).

Figure 3. Interindividual variability of the hand somatosensory representation in the MNI space. (A) Contralateral distribution of single subjects' representation of the right dorsum of the hand in S1. Brodmann classification was based on probabilistic cytoarchitectonic maps (JuBrain Anatomy Toolbox v3.0; Simon Eickhoff, Institut für Neurowissenschaften und Medizin, Forschungszentrum Jülich, Jülich, Germany). Bicolored data points indicate overlapping Brodmann Areas, depending on the z-coordinate in the transverse plane (see part (D)). (B) Detailed distribution over the left hemisphere. (C) Barycentre of the hand representation (shown in dots) on the left hemisphere with amplitude bars representing the dispersion (shown in lines). MNI barycentres of the hand representation on the left hemisphere $(x=-38$ (SE: \pm 4.3 , Range: $-45--27), y=-30.5$ (SE: \pm 4.3 , Range: $-40--22), \mathrm{z}=62$ (SE: \pm 5.0 , Range: $53-74)$ ). (D) Schematic representation of the anterior parietal areas BA3a, BA3b, BA1, and BA2, indicating that all data points lay within the postcentral gyrus based on a probabilistic atlas of human cortical brain areas (Harvard-Oxford macroanatomical atlas). 
Figure 4. Cortical surface mapping of functional somatosensory activations of the random effects general linear models of sensory-tactile stimulation of the clitoral region (left hemisphere: $\mathrm{x}=-18, \mathrm{y}=-34, \mathrm{z}=74 ; T=7.72$, $p_{\text {FWE-corr }}=0.024$; right hemisphere: $\left.\mathrm{x}=18, \mathrm{y}=-40, \mathrm{z}=68 ; T=10.26, p_{\text {FWE-corr }}<0.0001\right)$.

Figure 5. (A) Scatter plot with standard error (SE) on the correlation between frequency of sexual intercourse per week within the past 12 months and left-hemispheric genital field cortical thickness. Data points are plotted as residuals with correction for covariates. (B) Scatter plot with standard error (SE) on the correlation between frequency of sexual intercourse per week since onset of sexual contact and left-hemispheric genital field cortical thickness. Data points are plotted as residuals with correction for covariates. (Partial correlation values of covariates with genital field cortical thickness: Age: $\mathrm{r}=-.460, p=.055$; Years of sexual intercourse: $r=-.380, \mathrm{p}$ $=.120$; Whole brain cortical thickness: $\mathrm{r}=.309, p=.213$ ). 
Table 1. Characteristics of the Sample and Behavioral Data $(N=20)$

\begin{tabular}{|c|c|}
\hline Age, mean \pm SD & $23.10 \pm 4.35$ \\
\hline \multicolumn{2}{|l|}{ Ethnicity, n (\%) } \\
\hline European & $18(90 \%)$ \\
\hline Middle East & $1(5 \%)$ \\
\hline Asian & $1(5 \%)$ \\
\hline \multicolumn{2}{|l|}{ Education, $\mathbf{n}(\%)$} \\
\hline Enrolled in University & $20(100 \%)$ \\
\hline Bachelor degree completed & $6(30 \%)$ \\
\hline Master degree completed & $2(10 \%)$ \\
\hline \multicolumn{2}{|l|}{ Sexual Orientation $^{1}, \mathrm{n}(\%)$} \\
\hline Heterosexual & $17(85 \%)$ \\
\hline Bisexual & $3(15 \%)$ \\
\hline Homosexual & $0(0 \%)$ \\
\hline \multicolumn{2}{|l|}{ Partnership $^{1}$, n (\%) } \\
\hline Monogamous Partnership & $14(70 \%)$ \\
\hline Polygamous Partnership & $1(5 \%)$ \\
\hline No Partnership & $5(25 \%)$ \\
\hline \multicolumn{2}{|l|}{ Sexual Behavior ${ }^{1}$, mean \pm SD } \\
\hline Frequency of Sexual Intercourse/Week since Onset of Sexual Contact & $1.46 \pm 0.93$ \\
\hline Frequency of Sexual Intercourse/Week within the Past 12 Months & $1.91 \pm 1.30$ \\
\hline \multicolumn{2}{|c|}{ Perceived Pleasantness/Sexual Arousal during Sensory-Tactile Clitoral Stimulation ${ }^{1,2}$, mean $\pm \mathrm{SD}$} \\
\hline Pleasantness & $5.10 \pm 0.91$ \\
\hline Sexual Arousal & $4.00 \pm 1.41$ \\
\hline \multicolumn{2}{|l|}{ Contraception and Menstrual Cycle ${ }^{1}, \mathrm{n}(\%)$} \\
\hline Hormonal Contraception & $7(35 \%)$ \\
\hline Follicular Phase & $5(25 \%)$ \\
\hline Ovulation & $3(15 \%)$ \\
\hline Luteal Phase & $3(15 \%)$ \\
\hline Irregular Menstrual Cycle & $2(10 \%)$ \\
\hline \multicolumn{2}{|l|}{ Handedness ${ }^{1}, \mathrm{n}(\%)$} \\
\hline Right-handed & $18(90 \%)$ \\
\hline Left-handed & $2(10 \%)$ \\
\hline
\end{tabular}


Table 2. Individual and Group Cortical Activations in Response to Sensory-Tactile Stimulation of Clitoris or Dorsum of the Right Hand.

Coordinates indicate the somatosensory localizations in the $\mathrm{x}$ (mediolateral, with positive values for right hemisphere and negative values for left hemisphere), $\mathrm{y}$ (rostrocaudal, with negative values for caudal), and $\mathrm{z}$ (dorsoventral, with positive values for dorsal) axes in the MNI space. Individual and group activations were significant at $\mathrm{p}<0.001 \mathrm{without}$ correction or $\mathrm{p}<0.05$ with family-wise error (FWE) correction for multiple comparisons. ---, no functional activations detected.

\begin{tabular}{|c|c|c|c|c|c|c|c|c|c|c|c|c|}
\hline & $\begin{array}{l}\text { Genital } \\
\text { Representation } \\
\text { Left Hemisphere }\end{array}$ & & & & $\begin{array}{l}\text { Genital } \\
\text { Representation } \\
\text { Right Hemisphere }\end{array}$ & & & & $\begin{array}{l}\text { Hand } \\
\text { Representation } \\
\text { Left Hemisphere }\end{array}$ & & & \\
\hline $\begin{array}{l}\text { Single } \\
\text { Subject }\end{array}$ & $\begin{array}{l}\text { Center of gravity } \\
(x, y, z)\end{array}$ & $t$ value & $p$ threshold & $\begin{array}{l}\text { Cortical } \\
\text { Thickness }\end{array}$ & $\begin{array}{l}\text { Center of gravity } \\
(x, y, z)\end{array}$ & $t$ value & $p$ threshold & $\begin{array}{l}\text { Cortical } \\
\text { Thickness }\end{array}$ & $\begin{array}{l}\text { Center of gravity } \\
(x, y, z)\end{array}$ & $t$ value & $p$ threshold & $\begin{array}{l}\text { Cortical } \\
\text { Thickness }\end{array}$ \\
\hline 1 & $-21,-40,74$ & 14.48 & FWE $\quad 0.05$ & 2.3309 & $18,-40,80$ & 5.75 & FWE $\quad 0.05$ & 2.5585 & $-42,-37,59$ & 4.92 & FWE $\quad 0.05$ & 2.9968 \\
\hline 2 & $-24,-34,77$ & 3.35 & uncorr. 0.001 & 2.0890 & $15,-31,71$ & 2.26 & uncorr. 0.001 & 1.5174 & $-33,-31,68$ & 1.68 & uncorr. 0.001 & 2.4194 \\
\hline 3 & --- & -- & --- & -- & $15,-43,62$ & 3.22 & uncorr. 0.001 & 2.1214 & $-27,-31,68$ & 4.56 & FWE 0.05 & 2.4300 \\
\hline 4 & $-18,-40,62$ & 10.12 & FWE $\quad 0.05$ & 2.2791 & --- & --- & --- & --- & $-39,-34,65$ & 4.50 & FWE 0.05 & 2.3970 \\
\hline 5 & $-18,-46,68$ & 4.83 & uncorr. 0.001 & 2.1010 & --- & --- & --- & --- & $-39,-40,62$ & 2.70 & uncorr. 0.001 & 2.8083 \\
\hline 6 & $-21,-40,71$ & 3.01 & uncorr. 0.001 & 2.5363 & $18,-37,71$ & 6.00 & uncorr. 0.001 & 2.1836 & $-36,-28,65$ & 4.26 & FWE 0.05 & 1.7383 \\
\hline 7 & $-21,-34,80$ & 9.01 & FWE $\quad 0.05$ & 2.2215 & $27,-34,71$ & 17.29 & FWE 0.05 & 1.7881 & $-45,-31,62$ & 13.59 & FWE $\quad 0.05$ & 2.2301 \\
\hline 8 & $-21,-40,71$ & 14.13 & FWE $\quad 0.05$ & 2.4748 & $21,-37,71$ & 10.29 & FWE 0.05 & 2.7262 & $-39,-22,65$ & 3.81 & FWE $\quad 0.05$ & 2.1545 \\
\hline 9 & $-15,-34,71$ & 4.62 & uncorr. 0.001 & 2.3893 & $18,-37,65$ & 6.31 & uncorr. 0.001 & 2.0032 & --- & -- & --- & -- \\
\hline 10 & $-15,-31,65$ & 7.06 & uncorr. 0.001 & 2.4279 & $18,-40,74$ & 7.68 & uncorr. 0.001 & 2.6370 & $-36,-28,65$ & 2.68 & FWE $\quad 0.05$ & 2.1161 \\
\hline 11 & $-18,-40,68$ & 11.76 & FWE 0.05 & 2.6785 & $18,-34,74$ & 10.04 & FWE 0.05 & 2.0501 & $-36,-25,65$ & 8.55 & FWE $\quad 0.05$ & 2.3915 \\
\hline 12 & $-21,-37,77$ & 7.68 & uncorr. 0.001 & 2.4222 & $21,-37,68$ & 12.56 & uncorr. 0.001 & 2.1976 & $-42,-37,56$ & 7.05 & FWE $\quad 0.05$ & 2.3930 \\
\hline 13 & --- & -.- & --- & -- & $27,-37,71$ & 3.34 & uncorr. 0.001 & 2.2787 & $-42,-40,56$ & 5.38 & uncorr. 0.001 & 2.7626 \\
\hline 14 & $-15,-31,77$ & 4.52 & uncorr. 0.001 & 1.7867 & $12,-40,71$ & 10.24 & uncorr. 0.001 & 2.0809 & $-33,-28,62$ & 4.93 & uncorr. 0.001 & 1.5778 \\
\hline 15 & $-18,-37,68$ & 6.99 & FWE 0.05 & 2.2393 & $18,-40,80$ & 8.92 & FWE 0.05 & 2.3916 & $-45,-31,56$ & 2.57 & uncorr. 0.001 & 2.8190 \\
\hline 16 & $-21,-40,74$ & 6.46 & FWE $\quad 0.05$ & 2.0462 & $21,-34,77$ & 9.89 & FWE $\quad 0.05$ & 2.2819 & $-33,-34,53$ & 2.31 & uncorr. 0.001 & 1.9530 \\
\hline 17 & $-21,-43,71$ & 3.84 & uncorr. 0.001 & 2.8488 & $09,-43,68$ & 4.66 & uncorr. 0.001 & 2.3182 & $-45,-28,62$ & 3.21 & FWE 0.05 & 2.8617 \\
\hline 18 & $-18,-37,74$ & 6.46 & FWE 0.05 & 2.4128 & $18,-37,71$ & 7.80 & FWE 0.05 & 2.5398 & $-36,-28,59$ & 3.06 & uncorr. 0.001 & 1.6492 \\
\hline 19 & $-18,-37,74$ & 7.48 & FWE 0.05 & 1.8379 & $18,-40,71$ & 17.96 & FWE 0.05 & 2.0445 & $-39,-37,62$ & 7.24 & FWE $\quad 0.05$ & 2.1116 \\
\hline 20 & $-27,-40,71$ & 2.83 & uncorr. 0.001 & 2.6396 & $18,-40,71$ & 5.80 & uncorr. 0.001 & 2.3120 & $-36,-28,65$ & 2.25 & uncorr. 0.001 & 1.7425 \\
\hline \multirow[t]{2}{*}{ Group } & $\begin{array}{l}\text { Center of gravity } \\
(x, y, z)\end{array}$ & $t$ value & $p$ threshold & & $\begin{array}{l}\text { Center of gravity } \\
(x, y, z)\end{array}$ & $t$ value & $p$ threshold & & $\begin{array}{l}\text { Center of gravity } \\
(x, y, z)\end{array}$ & $t$ value & $p$ threshold & \\
\hline & $-18,-34,72$ & 7.72 & FWE $\quad 0.05$ & & $18,-40,68$ & 10.26 & FWE 0.05 & & $-33,-31,62$ & 6.13 & uncorr. 0.001 & \\
\hline
\end{tabular}




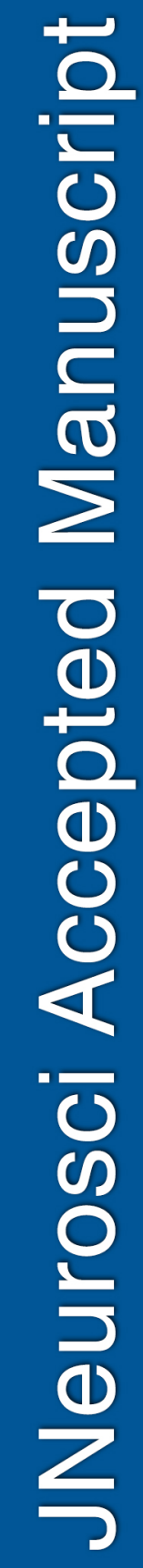

Figure 2.
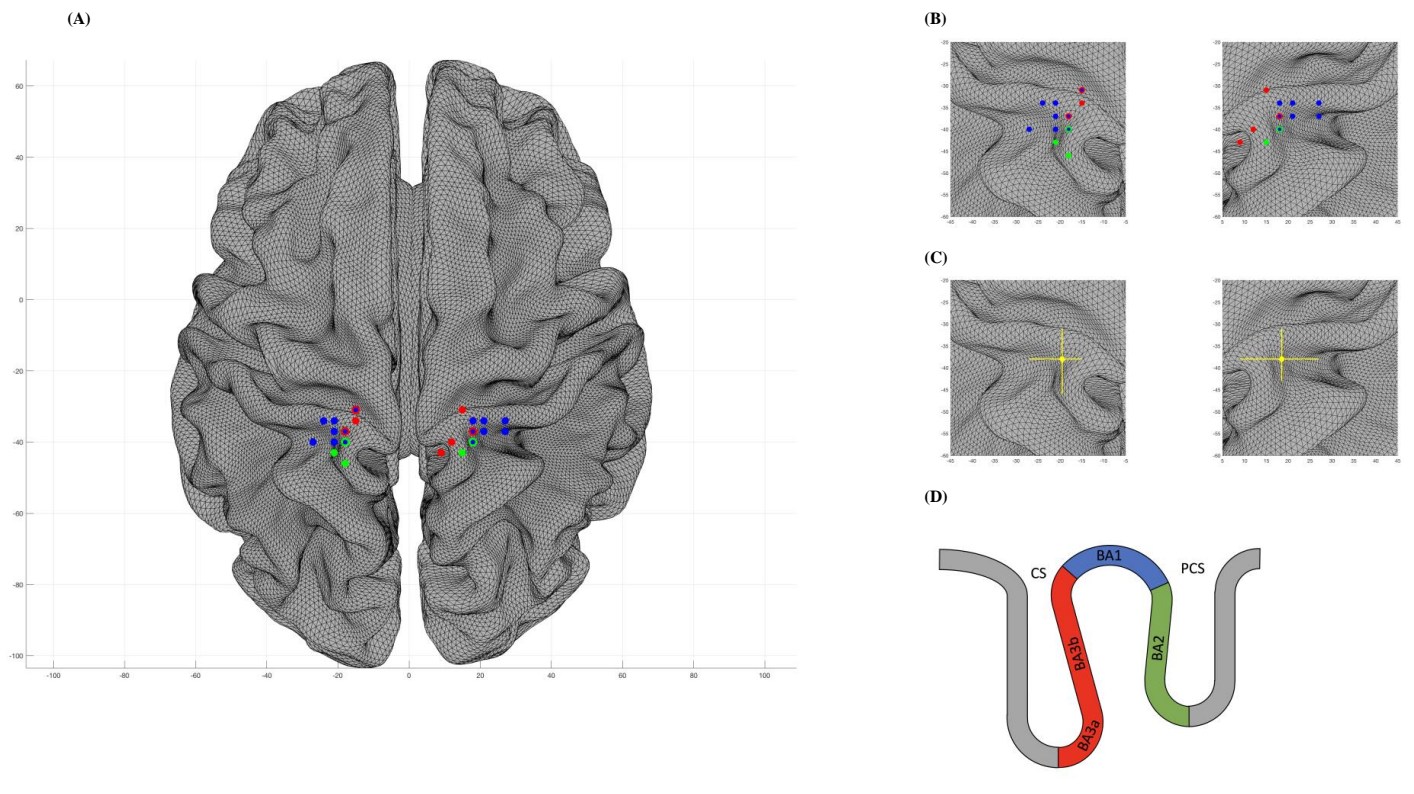
Figure 3.

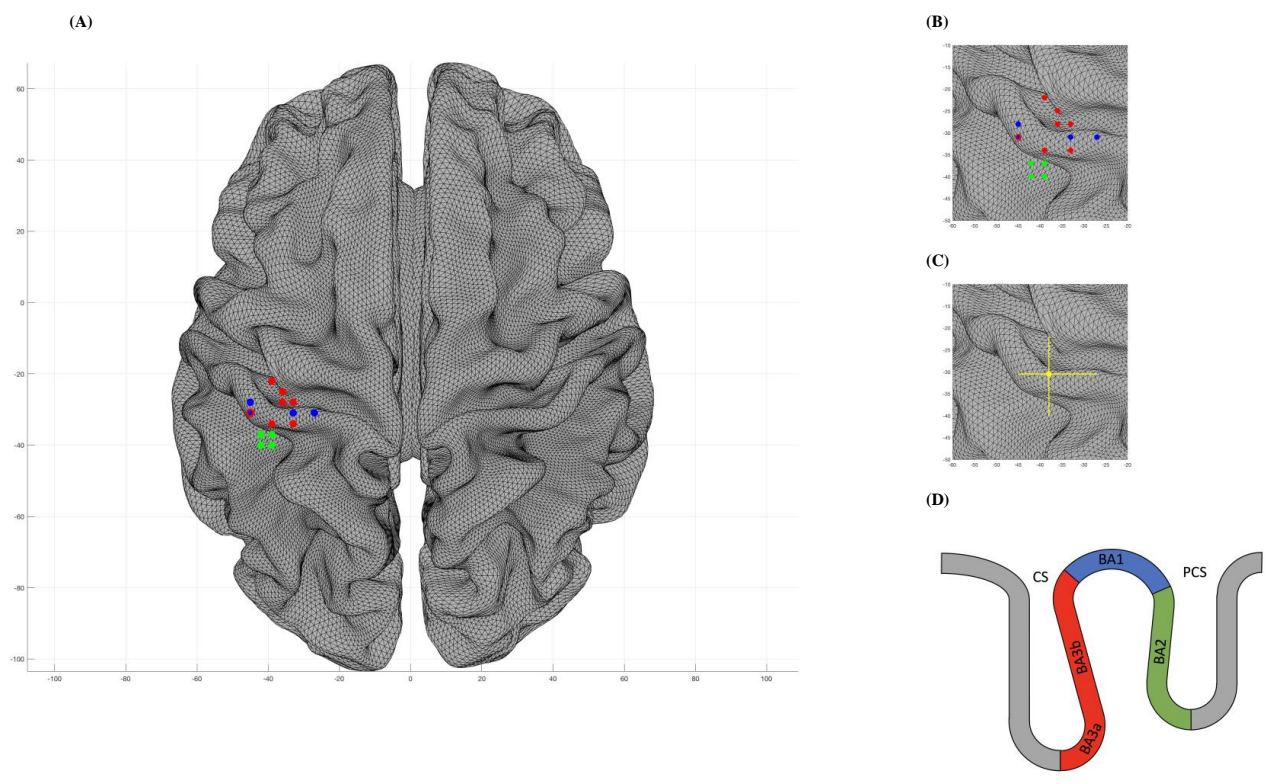

0

$\boldsymbol{O}$

O

3

(1)

2 


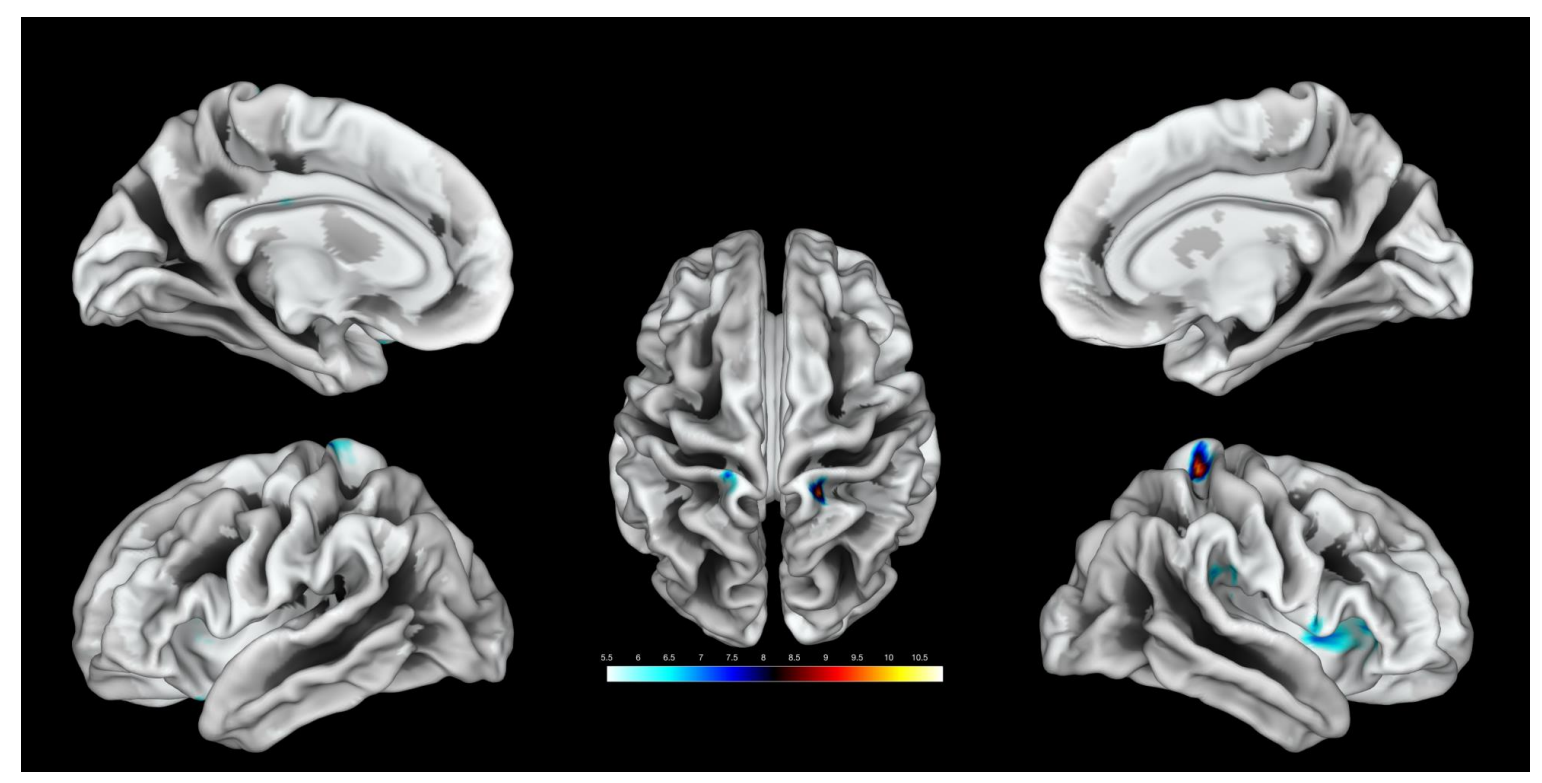


Figure 5.

(A)

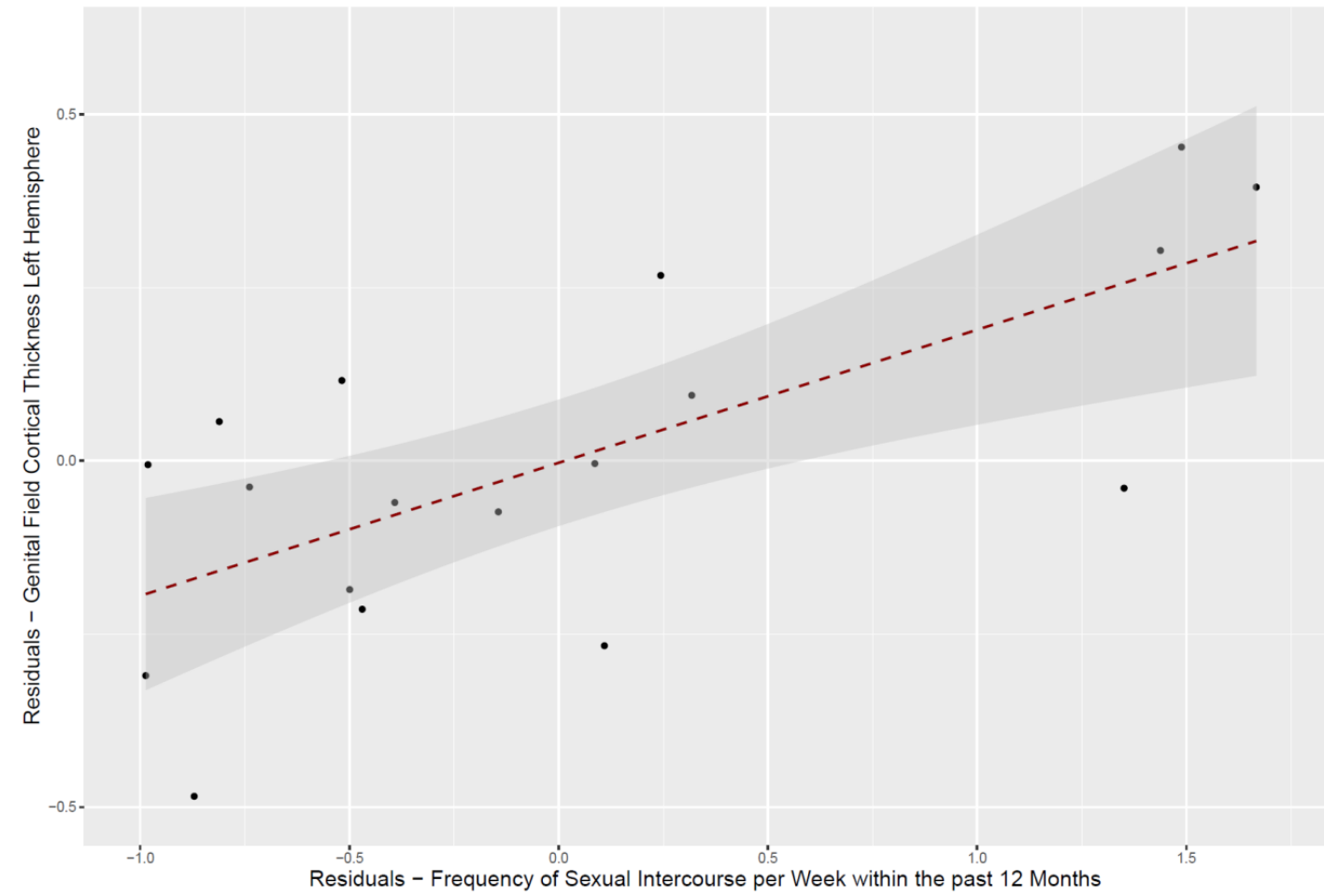

(B)

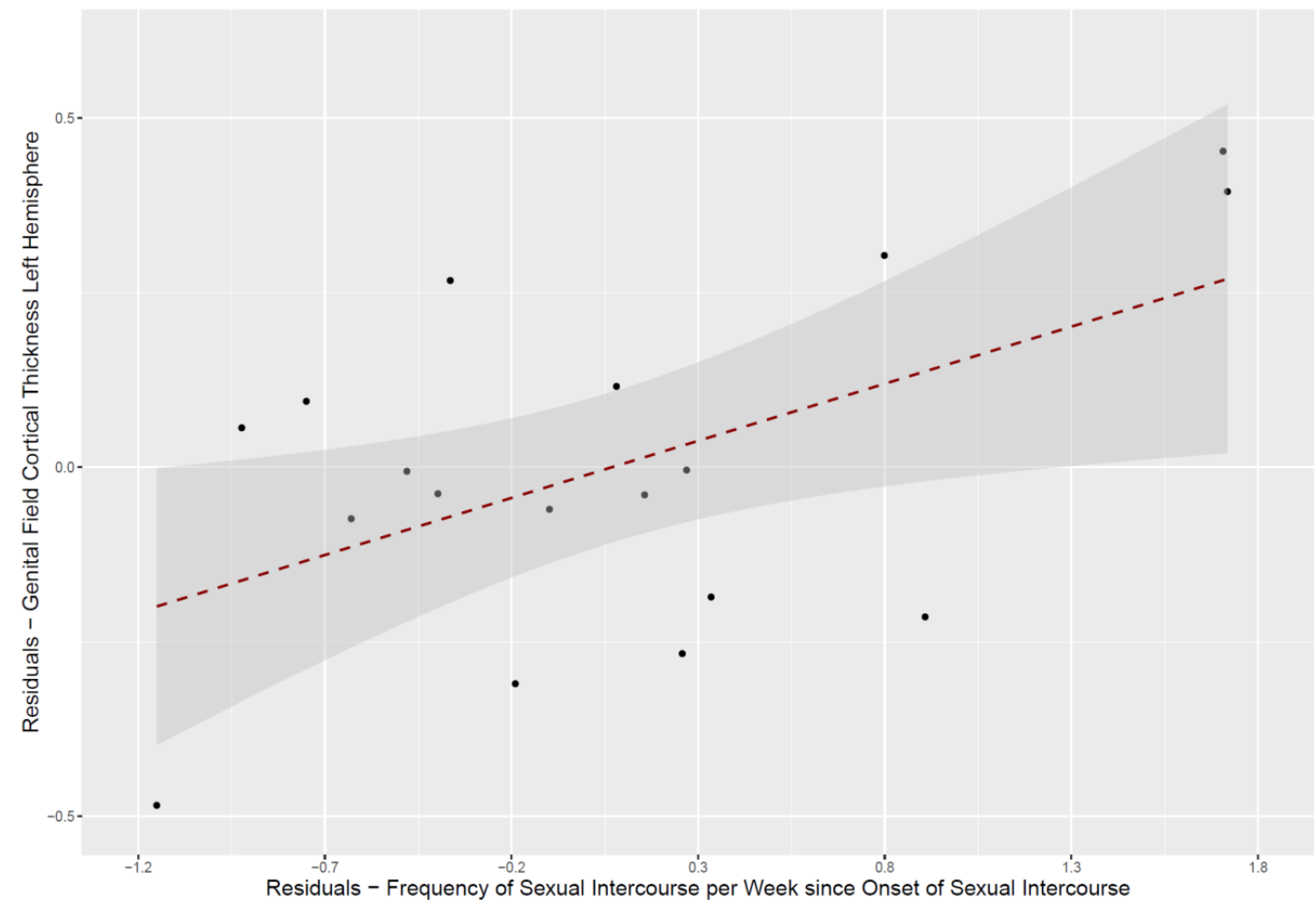

